\title{
"Maskandi Experience": Exploring The Use of A Cultural Song for Community Engagement in Preparation for A Pilot Sterile Insect Technique Release Programme for Malaria Vector Control in KwaZulu-Natal Province, South Africa 2019
}

Pinky Manana ( $\square$ pinky.manana@yahoo.com )

National Institute for Communicable Diseases https://orcid.org/0000-0002-6502-4308

\section{Sara Jewett}

University of the Witwatersrand

Jabulani Zikhali

Clinton Health Access Initiative

\section{Dumsani Dlamini}

university of the witwatersrand

\section{Nondumiso Mabaso}

university of the witwatersrand

\section{Zothile Mlambo}

University of the Witwatersrand Wartenweiler Library: University of the Witwatersrand

Roxanne Ngobese

University of the Witwatersrand

\section{Givemore Munhenga}

National institute for communicable diseases

\section{Research}

Keywords: sterile insect technique, malaria control, community engagement, cultural song, KwaZulu - Natal Province, South Africa

Posted Date: November 4th, 2020

DOI: https://doi.org/10.21203/rs.3.rs-100623/v1

License: @ (i) This work is licensed under a Creative Commons Attribution 4.0 International License. Read Full License

Version of Record: A version of this preprint was published at Malaria Journal on April 28th, 2021. See the published version at https://doi.org/10.1186/s12936-021-03736-9. 


\section{Abstract}

Background An assessment of the Sterile Insect Technique (SIT) as a complementary malaria vector control tool is at an advanced stage in South Africa. The technique involves the release of laboratory-reared sterilised male mosquitoes of the major malaria vector Anopheles arabiensis, raising social, ethical and regulatory concerns. Therefore, its implementation largely depends on community participation and acceptance. Against this background it is critical that robust and effective community strategies are developed. This study describes the development of a cultural song to engage the community and increase awareness on SIT and malaria control in KwaZulu-Natal, South Africa.

Methods An exploratory concurrent mixed-methods study was conducted to get opinions about the effectiveness of a cultural song developed to engage communities and increase acceptability of the SIT technology. Two self-administered surveys (expert and community) were conducted. Additionally, more in depth opinions of the song and its effectiveness in conveying the intended information were investigated through three community dialogue sessions with community members in the study area.

Results A total of 40 experts and 54 community members participated in the survey. Four themes were identified in relation to the appropriateness and effectiveness of the song, with a fifth theme focused on recommendations for adaptations. Overall, the song was well received with the audience finding it entertaining and informative. Responses to unstructured questions posed after the song showed an increase in the knowledge on malaria transmission and SIT technology. In particular, the explanation that male mosquitoes do not bite allayed anxiety and fears about the SIT technology.

Conclusion The song was deemed both culturally appropriate and informative in engaging community members about the SIT technology. It proved useful in promoting health messages and conveying SIT technology as a complementary malaria vector control tool. With minor adaptations, the song has potential as an area-wide community engagement tool in areas targeted for sterile male releases.

\section{Background}

Malaria is a public health challenge worldwide, with approximately 228 million cases and 405000 deaths reported in $2018^{1}$. More than $90 \%$ of malaria cases and deaths were reported from Africa, with sub-Saharan Africa being the most affected region $^{2}$. In 2018, the incidence of malaria was estimated at 57 cases per 1000 population ${ }^{1}$. The disease impacts negatively on the social, health and economic lives of affected people ${ }^{3}$. Although significant progress has been made in reducing the malaria burden in South Africa (SA), sporadic outbreaks still occur in the low-altitude northern and north-eastern regions of the country's three endemic provinces (Limpopo, Mpumalanga, KwaZulu-Natal (KZN)). In these endemic regions about 4.3 million people are at risk ${ }^{3,4}$.

Success in reduction of malaria transmission in SA is largely attributed to organised vector control achieved through Indoor residual spraying (IRS) of households with dicloro-diphenyl-trichloroethane (DDT) and pyrethroids. This approach has been in operation for over seven decades 5,6 . However, although IRS remains effective, it faces a number of challenges including insecticide resistance in targeted vector populations, outdoor biting populations that are not amenable to IRS, environmental concerns over continual use of insecticides and the high economic cost of using insecticides in low malaria transmission settings ${ }^{7,8}$. Against this background, additional vector control interventions are needed to supplement the current strategy if the South African government is to meet its mandate to eliminate malaria by $2023^{9}$. One such strategy under investigation is the Sterile Insect Technique (SIT) ${ }^{10}$. 
Feasibility studies on the applicability of SIT against the major malaria vector Anopheles arabiensis are ongoing ${ }^{11}$. Significant progress has been made in various aspects of the SIT packages including mass rearing, sterilization/mating compatibility and competitiveness, and development of sex separation systems ${ }^{6,11,12}$. This has paved the way for open field trials. To implement field trials successfully it is critical that cohesion between the community and the project is established. According to the $\mathrm{WHO}^{13}$, community engagement is central to any public health intervention. This is particularly so for the SIT technology that hinges on the release of laboratory-altered mosquitoes. The release of mosquitoes into communities raises social, ethical and regulatory concerns. Therefore, there is a need to characterise and robustly address such concerns before field trials can be initiated. It is important to engage and increase awareness about the programme in the target communities to ensure adequate cooperation and participation. Previous studies have demonstrated that directly engaging the community plays an important role in improving the acceptability and effectiveness of programmes ${ }^{10,14-17}$.

Community engagement is defined as working with all relevant partners who share the same interests to foster meaningful research; and collaboration to achieve common goals, including being sensitive to community contributions so as to protect their beliefs ${ }^{18}$. It involves supporting those affected to understand the risks they face, and empower them in making informed actions ${ }^{13}$. Community engagement efforts should be mindful of differences in culture, ethics, customs, and social structure between populations ${ }^{16,19}$. Any programmes that fails to consider beliefs and perceptions of the community face negative attitudes or practices ${ }^{16}$, cultural impositions ${ }^{20}$, introjection ${ }^{21}$, and symbolic violence ultimately leading to failure in achieving the intended goals ${ }^{10}$.

Several strategies for communication and engaging communities' are available. Different countries choose to use different methods based on the most effective strategies applicable under their local context. In sub-Saharan Africa, use of artistic forms ${ }^{22-25}$ to engage with communities is the commonly reported strategy. According to Bunn and colleagues $^{23}, 17$ different categories of art forms are widely used in sub-Saharan Africa with theatre, music and song, TV/radio, visual arts and storytelling being among the top 5 .

Music and song are used broadly, especially during advertising. Music significantly increases the ability of audiences to understand and remember a concept. This is often done through "jingles" and repetitions ${ }^{26}$. Previous studies ${ }^{27,28}$ have reported the use of music to promote behavioural change in adolescents and adults to help with disease prevention and management. Music, song and dance were used in Africa for HIV/ AIDS and Ebola promotional messages, to improve preventative interventions for these diseases ${ }^{24,29-36}$. During these community engagement campaigns, researchers either worked with popular artists or musicians to compose songs that were used or worked with communities to develop the songs. Specific to malaria, a song in Gambia was used successfully to encourage bed net repair for malaria prevention ${ }^{37}$. A study done in $\mathrm{SA}^{38,39}$ in Zulu-speaking communities reported that music provided support networks for people and a platform for preventive communication.

"Maskandi" is a type of traditional Zulu music that is popular and mostly played in KwaZulu-Natal (KZN) Province because of its richness in the Zulu heritage and importance to the Zulu tribe ${ }^{40}$. It has been in existence for many years and evolved within South African society. In an evaluation of how this indigenous music genre could be used as a tool for improving literacy, Ntombela's ${ }^{40}$ revealed a number of socio-cultural themes embedded in "Maskandi" music. These include use of metaphoric expressions, call-and-response patterns, repetition, indirection, interactive and group dynamics. These are general, yet particular, to the isiZulu tradition ${ }^{40}$. Against this background, "Maskandi" is a potential medium that can be used in (KZN) to engage the community for any new public health intervention. In this study the acceptability of using a cultural "Maskandi" genre song to engage with communities, increase awareness and knowledge on malaria control with specific focus on an area targeted for pilot of sterile male mosquito releases in Jozini, KZN, SA was assessed. 


\section{Methods}

\section{Study design}

The study used an exploratory concurrent mixed method that included both quantitative and qualitative approaches (Figure 1).

\section{Study setting}

The study was conducted in Mamfene, Jozini local municipality, KZN. Jozini local municipality is part of uMkhanyakude District Municipality and is located in northern KZN, sharing boarders with Swaziland and Mozambique (Figure 2). The Jozini local municipality is estimated to be $3442 \mathrm{~m}^{2}$. It is the most populated municipality within the UMkhanyakude district municipality with an estimated population of $198215^{41}$. The total number of households is 44584 . Jozini's population is young, with $65 \%$ of the people aged below 25 . The $0-14$ year olds constitute $41.5 \%$ of the population and $15-24$ years constitute $23 \%$ of the population. Adults aged 65 and above constitute only $3.4 \%$ of the total population. Females constitute $54 \%$ of the population.

The Mamfene area comprises 10 sections, three of which serve as SIT sentinel entomological surveillance sites. The population in the three sections is: section $2(n=2024)$; section $8(n=4592)$ and section $9(n=2167)^{41}$. The community of Mamfene uses three health care facilities. There is also one community hall, two churches, twenty-one secondary schools, five primary schools, four colleges and one community radio station.

\section{Song development}

A "Maskandi" group known in the community was approached to assist with composing a song on SIT technology. The group was provided with a pamphlet summarizing information on malaria and SIT technology as a guide to develop lyrics and music for the song in IsiZulu (local language). The translated lyrics can be found in Additional File 1. The song was first played during the World Malaria Day event commemorated in the study area in April 2019. The song was concurrently audio-recorded and used for data collection in this study.

\section{Study populations and sampling}

The primary population of interest for the study was the Mamfene community, which is the target area for a pilot sterile male release program. The campaign targeted two age groups; 18-49 year olds (considered as younger age group) and 50 years and above (considered as older age group). This was informed from a Malaria Knowledge, Attitudes and Practice (KAP) survey conducted in 2015 in Jozini ${ }^{42}$. The inclusion criteria were adults over 18 years old and residing in either Mamfene sections 2, 8 or 9, who were willing to participate and had given their consent to participate. To gather data, participants were invited to listen to a song followed by completion of a self-administered questionnaire. Furthermore, adults with the same inclusion criteria were also invited to community dialogue sessions.

The secondary study population was experts with experience in malaria control, who had an understanding of the local language and culture, including teachers in the study area. This included the malaria control programme teams, Environmental Health Practitioners, Information Education and Communication (IEC) teams, and Indunas (headsman). Lastly, medical scientists were recruited as experts based on their knowledge of science and understanding of the SIT technology.

\section{Questionnaire development}


The community questionnaire was divided into two sections: demographics and community engagement questions. The questionnaire was adapted from a tool used by Andersons et al. ${ }^{43}$. The questionnaire was translated into the study area local language (IsiZulu), and piloted with an IsiZulu speaking colleague at the National Institute for Communicable Diseases (NICD), originally from KZN. The piloted questionnaire was administered at different time points for participants in the different communities. The community survey had mixed (closed and open-ended) questions (Additional file 2) because there was a need for more information from the community regarding their perception, knowledge and understanding of the song.

The expert survey was structured similarly to the community survey, but was self-administered in English. The experts' survey used a 5-point Likert - scale to rank their opinions on the lyrics and the song (Additional file 3).

\section{Questionnaire administration}

The survey was self-administered by young participants, while interviewers supported the administration for older participants. Before questionnaire administration; project aims and study procedures were clearly explained and discussed with the participants. For both the community and expert surveys, an audio recording of the song was played once, for approximately 10 minutes, after which study participants completed the questionnaires. English subtitles were included for the experts as some of them were not conversant in IsiZulu.

\section{Data collection}

Data collection was done twice, first a community survey in November 2019 that was conducted immediately after a routine monthly community meeting with 100 community members attended and, secondly, an experts' survey done in January 2020.

For the community survey a total of 54 purposefully selected participants from the study area completed selfadministered questionnaires. Concurrently, interviewers administered a questionnaire to the older age groups or any participants who were struggling with completing the self-administered questionnaires. Immediately after completing the surveys, all community members were invited to stay for community dialogue sessions (CDS). The CDS were facilitated by the first author and trained field workers, using the questionnaire themes as a guide. These sessions were audio-recorded and field notes were taken. The CDS explored acceptability, cultural appropriateness of the song and the feasibility of using a song as an engagement strategy.

For the experts, information was collected through the self-administered questionnaire with 40 experts' participating in the survey.

\section{Data management and analysis}

Quantitative data were captured in RedCap and exported to Excel. Open response answers from the community survey were also exported from RedCap to an Excel file for post-hoc coding. Descriptive statistical analysis was conducted using STATA Version 15. Measures of central tendency and dispersion were calculated for quantitative variables and proportions were calculated for categorical variables. Frequency distribution tables were developed to show differences in the relative frequencies of variables.

Audio recordings of the CDS with the community members were downloaded from the tape recorder and stored on a secure office computer. Data were firstly transcribed verbatim and then translated from IsiZulu to English. The transcripts were saved in a Word file, where they were coded manually. A hybrid coding approach was conducted by the first two authors to establish themes as described by Fereday et $\mathrm{al}^{44}$. The steps include familiarisation of data by listening to individual audio recordings and reviewing of transcripts and field notes; reviewing and coding of sections of data 
transcripts using both inductive and deductive approaches matched to particular themes in the questionnaires, developing a working analytic framework, charting and summarising coded data in a table, analysis and interpretation of themes. Selected participants' quotes from each theme generated during analysis are presented.

As a final step, triangulation of both quantitative data and qualitative themes was conducted to identify any inconsistencies and to present a more integrated picture of the song's appropriateness.

\section{Results}

\section{Characteristics of study participants}

A total of 140 individuals participated in the study, 100 community members and 40 experts. Of the 100, 54 participated in the community survey and all (100) participated in the community dialogue sessions. The experts' survey had 40 participants. Their characteristics are summarized in Table 1.

The recruited $(n=54)$ community members completed the community survey and responded to all questions in the questionnaire. The majority of these participants were females $(69 \% ; n=37)$. Ages of the participants ranged between 18 and 78 years old, with a median age of 43 years. Heads of household accounted for $31 \%(n=17)$ of the participants. The majority of the participants $(68 \%, n=36)$ were unemployed and $65 \%(n=35)$ of the participants had some form of basic education. A total of $76 \%(n=41)$ participants were from section 9 (Ebiva), followed by section 8 with $15 \%(n=8)$ and section 2 with $9 \%(n=5)$.

All 100 community members participated in the community dialogue sessions. The age range of participants was between $18-78$ years with $69 \%$ being females. Participants included mothers, fathers, grandparents, youth and caregivers.

A total of 40 experts completed the self-administered questionnaire. Of these, $65 \%(n=26)$ were medical scientists, $27 \%$ $(n=11)$ were malaria control programme members and $8 \%(n=3)$ were educators. The majority of the participants were female $72 \%(n=29)$. The median age was 36 , with an interquartile range of $23-66$.

Four cross-cutting themes relating to the song's appropriateness were identified and explored during both the surveys and CDS. These included the song's cultural appropriateness, content appropriateness, appropriateness of delivery and overall appropriateness. Table 2 summarises individual questions relating to each of these themes and open-ended responses as well as CDS.

\section{Cultural appropriateness of song}

All community members (100\%) agreed that the song was culturally appropriate for Jozini community, as did $75 \%$ of the experts. In the CDS, many community members highlighted that the genre with 'an African beat' and use of isiZulu as elements made the song appropriate for intended use.

"We love this genre of music called "Maskandi" a lot and the song warned us about malaria." (Group 1, Female, 60)

"The song is perfect and having it in Isizulu is really helping in making us understand malaria. It is also good that the singer is a local resident." (Group 2, Female, 49)

On age appropriateness of lyrics in the song, all community members answered in the affirmative while $80 \%$ of experts agreed on the suitability of the lyrics for the targeted age groups. However, there were mixed views during CDS. Some opinions aligned with the survey: 
"This song is appropriate for all age groups and it warns us about the signs and symptoms of malaria." (Group 2, Female, 49)

However, other community members thought the song genre was more attractive to older listeners. One 26-year old female community member from Group 1 explained that it was less the content than the genre she found inappropriate:

"The song is very informative and warns us about malaria. However, I do not enjoy this type of music."

This opinion was however not shared by all young people, with a 28 year old female from Group 3 exclaiming: "This song has great rhythm and I can dance to it."

\section{Appropriateness of song delivery}

A variety of questions were asked regarding the song format. Over $95 \%$ of community members reported that the words were clear and easy to understand, that the message was easy to remember and that the use of repetition supported engagement. While community feedback was mainly positive, a few people highlighted issues around the aesthetic delivery of the song. For instance, one participant complained:

"The song is not clear enough. I did not get everything that the song is about, especially the backing vocalists." (Group 1, Female, 32)

Most negative comments about the song delivery focused on audibility, particularly towards the end of the song.

While the majority of experts gave positive responses regarding the delivery of the song, $85 \%$ of experts responded in the affirmative to all the three questions on song delivery, while there were more who expressed concern on the delivery of the song when compared to community members. One expert concluded, "The song is good, easy to understand, affirmative and appropriate." (Scientist and MCP staff) However, another expert expressed frustration: "The song is too long, too much repetition." (MCP staff)

This concern was however not echoed by any of the community members.

\section{Appropriateness of content}

In summary, $92 \%$ of all participants (both experts and community members combined) agreed that the malaria content and the SIT information in the song was useful and adequate.

To further explore content effectiveness, community comments were analysed using open-ended questions to determine what participants remembered from the song and what they learned. These results were coded, grouped and quantified (see Table 3). This data supplemented information obtained from the six closed-ended questions that were posed to both experts and community members about the song content.

Three strong themes about malaria emerged when the community was probed on what they learned from the song. These were: malaria is fatal but treatable, male mosquitoes do not bite and the morphological difference between a male and female mosquito. Of these, the message that female mosquitoes transmit pathogens that cause infections was the most remembered theme (33\%), followed by the fact that malaria can kill but is treatable (24\%). Malaria signs and symptoms were least remembered, with fewer than $10 \%$ remembering this thematic area. One expert (MCP staff, Male 38) summed this by recommending: "Expand on what is malaria and signs and symptoms."

The inclusion of information on mosquitoes being vectors for malaria was applauded by $97 \%$ of the experts. Repeated emphasis on the point that male mosquitoes do not bite in the song was hailed by $85 \%$ of the experts (Table 2 ), with $11 \%$ 
of community members spontaneously recalling this point (Table 3). This critical message was cited as a lesson learnt by $24 \%$ of the community members surveyed. About $18 \%$ participants from the community specified that they have learned about the difference in behaviour between male and female mosquitoes through the song, with $33 \%$ reporting that the song reminded them that only female mosquitoes transmit malaria (Table 3).

These views were also consistent with those expressed during CDS. In the CDS, participants expressed knowledge acquisition. One female participant summed:

"I gained a lot more information about malaria that I did not know before, including that only female mosquito transmit infection." (Female 61 and Male 49)

The inclusion of SIT as an additional vector control method in the song was applauded by $87 \%$ of experts (Table 2 ). About $80 \%$ of the experts surveyed thought mentioning that SIT also targets outdoor mosquitoes was appropriate and that the song had enough information to help people understand the SIT technology. A higher proportion of community (96\%) members also shared the same views. By contrast, when it came to retaining this information, fewer than $10 \%$ of the community remembered or reported learning about SIT in the open-ended questions.

In CDS, one participant noted:

"I have learned that male mosquito will be sterilized and released back into the wild, to help reduce the spread of malaria." (Female 40)

\section{Overall appropriateness}

Most experts (90\%) and all community members (100\%) agreed that the song was appropriate to engage the community about SIT.

In the CDS, participants noted:

"The song can really help communities and schools with malaria awareness and engagement. It is also useful to know that only female mosquito transmit infection." (Female 36)

"This song should be played on national radio for everyone to understand and hear about the SIT project." (Male 49)

The participants emphasized that this song should be played at schools, churches and the community, so that everyone can know and understand malaria and the SIT project, especially the young children. When asked if there was anything they could change about the song, all those who responded to this question (12\%) agreed with this recommendation: "Tone down the guitars so that back-up singers can be heard better" (Females, ages 18 - 40).

\section{Discussion}

Appropriateness is a multi-dimensional phenomenon. In this work appropriateness was taken in the context of whether a cultural song was acceptable and effective in conveying intended messages. Against this background, exploratory concurrent mixed methods were used to investigate the acceptability of using a cultural song in the "Maskandi" genre in three communities from Jozini, KwaZulu-Natal Province that fall within an area targeted for piloting the SIT technology. A local artist residing in the area was identified and worked with project members to generate or composition a song that will be used for community engagement purposes.

The findings of the study show that the community (both from the survey and community dialogue sessions) strongly agreed that the song was appropriate for age and culturally acceptable to engage and educate communities on malaria 
and the SIT technology. The acceptability of using music to convey information has been noted in similar interventions, accounting in part for its widespread use in Africa $24,27,36-39,28-35$. Overall participants' response was positive; the song was well received and the audience reported that it was informative and entertaining. They were more pleased that one of their own (local artist) composed the song and the genre was the one that spoke to their tradition and reminded them of where they come from. This is in agreement with Ntombela ${ }^{40}$ who reported on the value Zulu people place on the "Maskandi" genre of music. This approach of using popular artists and working with the communities is commonly used in Africa, ${ }^{29,34-41}$ and increases the impact music has and ownership by the locals as observed from this study.

The community members further reported that the song was easy to remember, it allowed them to engage and that they benefitted from the repetition of verse. This finding is in agreement with Allan ${ }^{26}$ that targeted songs or "jingles" can significantly increase audience ability to remember and understand the message.

The question of what was remembered and with what accuracy is critical to understanding how the audience engage with music. This is a key principle of audience reception research, which seeks to establish what kind of meanings audiences derive from communication and whether, based on their circumstances, these meanings have any effects (cognitive, social, political and/or emotional) ${ }^{45}$. One focus of this study was on what meaning audiences took from the song. The themes participants recalled, particularly about the links between mosquitoes and malaria and the differences between male and female mosquitoes, are promising, as the SIT project will be based on the mass release of sterile male mosquitoes. An understanding of these nuances may improve ultimate acceptability of the pilot project. A 2015 study by Manana et $\mathrm{al}^{42}$ found that $37 \%$ of study participants in KZN associated female mosquitoes as the carriers of infection. Our current study suggests that exposure to the "Maskandi" song may increase these knowledge levels ${ }^{37-39}$.

Although knowledge that malaria can kill if not treated was spontaneously remembered by at least a quarter of the community participants and was the strongest theme, recall of malaria signs and symptoms was poor. In contrast, a knowledge, attitudes and practices (KAP) study by Manana and colleagues in the same communities reported $100 \%$ of the participants remembered that malaria can kill if untreated and $63 \%$ were able to recall at least three or four symptoms for malaria ${ }^{42}$. The difference in results could be that this study post-coded open-ended questions whereas the KAP study used a quantitative survey, where the survey questions themselves may have prompted them to remember.

The song also emphasized that the SIT technology is not replacing the current vector control method (indoor residual spraying), but is an additional tool to compliment the current malaria control strategy ${ }^{10}$. Only $10 \%$ spontaneously reported to have remembered and learned about the SIT project. This finding is similar to the Manana et al ${ }^{42}$ study. More effort needs to be dedicated to improving awareness on the SIT technology through additional communication channels. This is important, as communities are still expected to comply with the national requirements regarding IRS as it is currently the main vector control strategy in South Africa ${ }^{5,6}$.

More practically, based on participant feedback, alterations can be made to the song to improve its effectiveness. The main recommendation focused on the audibility of the sound. It was recommended by some members of the community and the expert group that the song could be shortened and guitar sounds reduced so that the back-up voices could be heard well.

The study is not without limitations. As the survey sampling was not random across communities, the findings cannot be generalised. The qualitative component was done through community dialogues with large numbers of participants of mixed ages and genders, which may have resulted in some people feeling uncomfortable to share their views. Poor sound quality in the CDS recordings may have led to a loss of information during translation from isiZulu to English, or loss of meaning if note-takers missed details about the context of the dialogues. In retrospect, the use of focus group 
discussions, segmented by age and gender, could have mitigated these risks. Nevertheless, the use of mixed methods was a strength, as multiple sources of data enabled triangulation of results.

While the "Maskandi" experience is promising, whether increased knowledge about SIT will be sufficient to result in

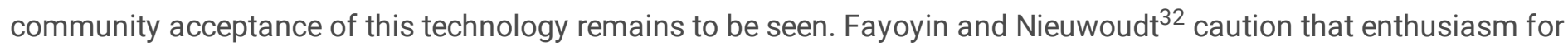
music as an enabler of change needs to be supported by empirical evidence, which is often lacking. As such, we recommend that provisions are made for an impact evaluation of whether this and other efforts to engage with the Jozini communities results in greater community support for SIT.

\section{Conclusion}

The "Maskandi" song developed was deemed educational, entertaining, culturally appropriate and informative in engaging community members on the SIT technology and its potential as a complementary vector control tool. We conclude that cultural music is a potential tool that supports community engagement and if complimented with additional communication channels can reinforce and supplement the dissemination of key information. The recommendations made regarding the song will be considered and the current version updated and re-recorded as part of an ongoing effort to engage communities about SIT in KZN.

\section{Declarations}

\section{Ethical approval and consent to participate}

Ethical review and approval were granted by the Faculty of Health Sciences Research Ethics Committee of the University of the Witwatersrand (CR 20200218-10/ AREC-101210-002) and KwaZulu-Natal Health Research and Knowledge Management (KZ_202003_016). Written consent was given by all participants.

\section{Consent for publication}

Not applicable

\section{Availability of data and materials}

All data is available on reasonable request.

\section{Competing interest}

The authors declare that they have no competing interests

\section{Funding}

The study was funded by the International Atomic Energy Agency (IAEA) under their Technical Cooperation Programme (SAF 5014/5017). The work was supported by the Department of Science and Innovation through their Health Innovation Scheme and partly by the National Research Foundation (Grant number 119765). We also acknowledge the support from ANTI-VeC Pump-Priming Grant AV/PP0027/1 from University of Glasgow.

\section{Author's contribution}

PNM conceived the study, designed the study tools, conducted interviews, analysed the data, and wrote the first and subsequent drafts of the manuscript. SJ analysed the data and contributed to writing of the manuscript. JZ, DD, NM, ZM, 
RN conducted interviews, GM participated in the conception of the study, supervised the work and contributed to the subsequent interpretation and writing of the manuscript. All authors read and approved the final manuscript.

\section{Acknowledgements}

The authors would like to acknowledge the KwaZulu-Natal Department of Health, Vector Control Laboratory staff and students particularly the SIT project personnel and the community in Jozini for allowing us to conduct the survey, discussions and all their support in this study. We are also grateful to Prof Basil Brooke for providing scientific writing advice and critically reviewing this manuscript.

\section{References}

1. Global Malaria Programme: WHO Global. World malaria report 2019 [Internet]. WHO Regional Office for Africa. 2019. Available from: https://www.who.int/news-room/fact-sheets/detail/malaria

2. Moonasar D, Morris N, Kleinschmidt I, Maharaj R, Raman J, Mayet NT, et al. F ROM CONTROL TO ELIMINATION What will move malaria control to elimination in South Africa? 2013;103(10):801-6.

3. Mabaso MLH, Sharp B, Lengeler C. Historical review of malarial control in southern African with emphasis on the use of indoor residual house-spraying. Trop Med Int Heal. 2004;9(8):846-56.

4. Maharaj R, Raman J, Morris N, Moonasar D, Durrheim DN, Seocharan I, et al. Epidemiology of malaria in South Africa: From control to elimination. South African Med J. 2013;103(10):779-83.

5. Klassen W. Introduction: development of the sterile insect technique for African malaria vectors. Malar J. 2009;8 Suppl 2:11.

6. Munhenga G, Brooke BD, Chirwa TF, Hunt RH, Coetzee M, Govender D, et al. Evaluating the potential of the sterile insect technique for malaria control: relative fitness and mating compatibility between laboratory colonized and a wild population of Anopheles arabiensis from the Kruger National Park, South Africa. Parasit Vectors. 2011;4(1):208.

7. Munhenga G, Brooke BD, Chirwa TF, Hunt RH, Coetzee M, Govender D, et al. Evaluating the potential of the sterile insect technique for malaria control: relative fitness and mating compatibility between laboratory colonized and a wild population of Anopheles arabiensis from the Kruger National Park, South Africa. Parasit Vectors [Internet]. 2011 Oct 31 [cited 2019 Jan 30];4(1):208. Available from:

http://parasitesandvectors.biomedcentral.com/articles/10.1186/1756-3305-4-208

8. World Health Organisation. Malaria elimination: a field manual for low and moderate endemic countries. Geneva World Heal Organ [Internet]. 2007;98. Available from: http://whqlibdoc.who.int/publications/2007/9789241596084_eng.pdf\%5Cnhttp://scholar.google.com/scholar? $\mathrm{hl}=$ en\&btnG=Search\&q=intitle:Malaria+Elimination:+A+field+manual+for+low+and+moderate+endemic+countries\#0

9. World Health Organization, World Health Organization. Global Malaria Programme. Global technical strategy for malaria, 2016-2030. WHO Geneva. 2015.

10. Alphey L, Benedict M, Bellini R, Clark GG, Dame D a, Service MW, et al. Sterile-Insect Methods for Control of Mosquito-Borne Diseases:An Analysis. Vector-Borne Zoonotic Dis. 2010;10(3):295-311.

11. Munhenga G, Brooke BD, Spillings B, Essop L, Hunt RH, Midzi S, et al. Field study site selection, species abundance and monthly distribution of anopheline mosquitoes in the northern Kruger National Park, South Africa. Malar J [Internet]. 2014 Jan 24 [cited 2019 Jan 30];13(1):27. Available from: http://malariajournal.biomedcentral.com/articles/10.1186/1475-2875-13-27

12. Munhenga G, Brooke BD, Gilles JRL, Slabbert K, Kemp A, Dandalo LC, et al. Mating competitiveness of sterile genetic sexing strain males (GAMA) under laboratory and semi-field conditions: Steps towards the use of the Sterile Insect 
Technique to control the major malaria vector Anopheles arabiensis in South Africa. Parasit Vectors [Internet]. 2016 Dec 2 [cited 2019 Jan 29];9(1):122. Available from: http://www.parasitesandvectors.com/content/9/1/122

13. WHO COMMUNITY ENGAGEMENT FRAMEWORK FOR QUALITY, PEOPLE-CENTRED AND RESILIENT HEALTH SERVICES [Internet]. 2017 [cited 2020 Sep 7]. Available from: http://apps.who.int/bookorders.

14. Oliva CF, Vreysen MJB, Dupé S, Lees RS, Gilles JRL, Gouagna LC, et al. Current status and future challenges for controlling malaria with the sterile insect technique: Technical and social perspectives. Acta Trop [Internet]. 2014;132(1):S130-9. Available from: http://dx.doi.org/10.1016/j.actatropica.2013.11.019

15. Kaneko A, Taleo G, Kalkoa M, Yamar S, Kobayakawa T, Björkman A. Malaria eradication on islands. Lancet. 2000 Nov 4;356(9241):1560-4.

16. Manderson L. Applying medical anthropology in the control of infectious disease. Vol. 3, Tropical Medicine and International Health. 1027.

17. Campbell C, Jovchelovitch S. Health, community and development: towards a social psychology of participation. J Community Appl Soc Psychol. 2000;10(4):255-70.

18. Joubert G (Georgina), Ehrlich R, Katzenellenbogen JM, Abdool Karim SS. Epidemiology : a research manual for South Africa [Internet]. Oxford University Press Southern Africa; 2007 [cited 2019 May 29]. 354 p. Available from: https://research-repository.uwa.edu.au/en/publications/epidemiology-a-research-manual-for-south-africa

19. Dyck VA, Hendrichs JP RA. The sterile insect technique: Principles and practices in area - wide intergrated pest management. 2nd editio. Springer; 2005.

20. Reid H, Vallely A, Taleo G, Tatem AJ, Kelly G, Riley I, et al. Open Access RESEARCH Baseline spatial distribution of malaria prior to an elimination programme in Vanuatu [Internet]. Vol. 9, Malaria Journal. 2010. Available from: http://www.malariajournal.com/content/9/1/150

21. Re-emergence of malaria in India - PubMed [Internet]. [cited 2020 Sep 7]. Available from: https://pubmed.ncbi.nlm.nih.gov/8926025/

22. Playing for Life: Performance in Africa in the Age of AIDS - ProQuest [Internet]. [cited 2020 Sep 7]. Available from: https://search.proquest.com/openview/0f81539e19b5d1428d0c1ae9a5dcccf4/1?pq-origsite=gscholar\&cbl=48187

23. Bunn C, Kalinga C, Mtema O, Abdulla S, DIllip A, Lwanda J, et al. Arts-based approaches to promoting health in subSaharan Africa: A scoping review. BMJ Glob Heal. 2020;5(5):1-15.

24. Stone RM. "Ebola in town": Creating musical connections in liberian communities during the 2014 crisis in West Africa. Afr Today. 2017 Mar 1;63(3):78-97.

25. McConnell BB. Music and health communication in The Gambia: A social capital approach. Soc Sci Med. 2016 Nov 1;169:132-40.

26. Allan D. Effects of popular music in advertising on attention and memory. J Advert Res. 2006;46(4):434-44.

27. Kim J, Wigram T, Gold C. The effects of improvisational music therapy on joint attention behaviors in autistic children: A randomized controlled study. J Autism Dev Disord [Internet]. 2008 Oct [cited 2020 Sep 7];38(9):1758-66. Available from: https://pubmed.ncbi.nlm.nih.gov/18592368/

28. Mcferran KS, Shoemark H, Fellow HS. How musical engagement promotes well-being in education contexts: The case of a young man with profound and multiple disabilities. [cited 2020 Sep 7]; Available from:

http://dx.doi.org/10.3402/qhw.v8i0.20570

29. Isabirye J. Philly lutaaya: Popular music and the fight against HIV/AIDS in Uganda. J Postcolonial Writ [Internet]. 2008 [cited 2020 Sep 7];44(1):29-35. Available from:

https://www.tandfonline.com/doi/abs/10.1080/17449850701820632

30. Bastien S. Reflecting and shaping the discourse: The role of music in AIDS communication in Tanzania. Soc Sci Med. 2009 Apr 1;68(7):1357-60.

Page $12 / 17$ 
31. Banda F, Mambwe K. Fighting hiv/aids through popular zambian music. Muziki [Internet]. 2013 [cited 2020 Sep 7];10:1-12. Available from: https://www.tandfonline.com/doi/abs/10.1080/18125980.2013.852738

32. A F. The Power of Song in the Struggle for Health and Development Outcomes in Africa: Lessons for Social and Behaviour Change Programmes. J Mass Commun Journal. 2017;07(04).

33. Frishkopf M. Popular music as public health technology: Music for global human development and "giving voice to health" in Liberia. J Folk Res. 2017 Jan 1;54(1-2):41-86.

34. Mcconnell BB, Darboe B. Music and the Ecology of Fear: Kanyeleng Women Performers and Ebola Prevention in The Gambia. Afr Today. 63(3).

35. Rivera M. Music, media, and the ethnopoetics of two Ebola songs in liberia. Afr Today. 2017 Mar 1;63(3):62-76.

36. Mobilizing Musical Performance and Expressive Culture in the Ebola 2014 Epidemic: Introduction on JSTOR [Internet]. [cited 2020 Sep 7]. Available from: https://www.jstor.org/stable/10.2979/africatoday.63.3.01

37. Panter-Brick C, Clarke SE, Lomas H, Pinder M, Lindsay SW. Culturally compelling strategies for behaviour change: A social ecology model and case study in malaria prevention. Soc Sci Med. 2006 Jun 1;62(11):2810-25.

38. Black SP. The intersubjective space-time of a Zulu choir/HIV support group in global perspective. Soc Semiot [Internet]. 2014 [cited 2020 Aug 29];24(4):381-401. Available from: https://www.tandfonline.com/doi/abs/10.1080/10350330.2014.929387

39. Black SP. The Morality of Performance: HIV Disclosure in Speech and Song in South Africa. Ethos [Internet]. 2015 Sep 1 [cited 2020 Sep 7];43(3):247-66. Available from: http://doi.wiley.com/10.1111/etho.12090

40. Ntombela SA. Maskandi: A critical discourse analysis of indigenous isiZulu songs. South African Linguist Appl Lang Stud [Internet]. 2016 Jun 30 [cited 2020 Aug 29];34(2):109-20. Available from:

https://www.tandfonline.com/doi/abs/10.2989/16073614.2016.1194219

41. Statistics South Africa. South African Statistics 2012 [Internet]. 2012. 1-201 p. Available from: http://www.localgovernment.co.za/locals/view/93/Jozini-Local-Municipality\#resources

42. Manana PN, Kuonza L, Musekiwa A, Mpangane HD, Koekemoer LL. Knowledge, attitudes and practices on malaria transmission in Mamfene, KwaZulu-Natal Province, South Africa 2015. BMC Public Health. 2017;18(1).

43. Anderson CM, McCrindle CME, Kruger T, McNeill F. Using participatory risk analysis to develop a song about malaria for young children in Limpopo Province, South Africa. Malar J [Internet]. 2018 Dec 27 [cited 2019 May 29];17(1):181. Available from: https://malariajournal.biomedcentral.com/articles/10.1186/s12936-018-2320-7

44. Fereday J. Demonstrating Rigor Using Thematic Analysis: A Hybrid Approach of Inductive and Deductive Coding and Theme Development. 2006;80-92.

45. Staiger J. Media reception studies. Media Reception Studies. 2005.

46. Ingabire CM, Alaii J, Hakizimana E, Kateera F, Muhimuzi D, Nieuwold I, et al. Community mobilization for malaria elimination: application of an open space methodology in Ruhuha sector, Rwanda. Malar J [Internet]. 2014 Dec 2 [cited 2019 May 28];13(1):167. Available from: https://malariajournal.biomedcentral.com/articles/10.1186/14752875-13-167

\section{Tables}


Table 1

Demographic characteristics of study participants during the assessment of acceptability of a cultural song as a community engagement medium in KwaZulu-Natal Province, South Africa.

\begin{tabular}{|llll|}
\hline Characteristics of study participants & Expert survey $\mathbf{N = 4 0 ( \% )}$ & Community survey & Community dialogues \\
\hline Age (in years) & & $\mathbf{N}=\mathbf{5 4}(\%)$ & $\mathbf{N}=\mathbf{1 0 0}(\%)$ \\
\hline Median age & $36(23-66)$ & $43(18-78)$ & $43(18-78)$ \\
\hline Gender & & & \\
\hline Female & $29(72)$ & $37(69)$ & $69(69)$ \\
\hline Position/ Role & & & \\
\hline Scientist & $26(65)$ & N/A & N/A \\
\hline Malaria control programme & $11(27)$ & N/A & N/A \\
\hline Educator & $3(8)$ & N/A & $2(2)$ \\
\hline Induna (headsman) & N/A & N/A & $40(40)$ \\
\hline Heads of households & N/A & $17(31)$ & $47(47)$ \\
\hline Unemployed & N/A & $36(67)$ & $60(60)$ \\
\hline Some form of education & N/A & $35(65)$ & \\
\hline
\end{tabular}


Expert and community responses to survey questions categorised by themes during the assessment of acceptability of a cultural song as a community engagement medium in KwaZulu-Natal Province, South Africa.

\begin{tabular}{|c|c|c|}
\hline Themes & $\begin{array}{l}\text { Experts } \\
\text { survey } \\
n=40 \text { (\%) }\end{array}$ & $\begin{array}{l}\text { Community } \\
\text { survey } \\
\mathrm{n}=54(\%)\end{array}$ \\
\hline \multicolumn{3}{|l|}{ Cultural Appropriateness } \\
\hline 1. Are the words in the song culturally appropriate for Jozini? & $30(75)$ & $54(100)$ \\
\hline 2. Are the words in the song age appropriate for Jozini? & $32(80)$ & $53(98)$ \\
\hline \multicolumn{3}{|l|}{ Delivery Appropriateness } \\
\hline 3. Are the words in the song clear and is it easy to understand what is being said? & $34(85)$ & $52(96)$ \\
\hline 4. Is the song easy to remember and does it allow people to engage? & $34(85)$ & $53(98)$ \\
\hline 5. Is repetition of verses in the song useful and can people benefit from it? & $34(85)$ & $54(100)$ \\
\hline \multicolumn{3}{|l|}{ Content Appropriateness } \\
\hline 6. Is information on malaria in the song useful and adequate? & $37(92)$ & $50(92)$ \\
\hline $\begin{array}{l}\text { 7. Is informing people that mosquitoes are the vector for malaria an appropriate } \\
\text { way to engage? }\end{array}$ & $39(97)$ & $\mathrm{n} / \mathrm{a}$ \\
\hline 8. Is informing people that male mosquitoes do not bite a useful way to engage? & $34(85)$ & $\mathrm{n} / \mathrm{a}$ \\
\hline $\begin{array}{l}\text { 9. Is informing people that SIT is an additional vector control method appropriate } \\
\text { and useful? }\end{array}$ & $35(87)$ & $\mathrm{n} / \mathrm{a}$ \\
\hline $\begin{array}{l}\text { 10. Is informing people that SIT will help target outdoors mosquitoes appropriate } \\
\text { and useful? }\end{array}$ & $32(80)$ & $\mathrm{n} / \mathrm{a}$ \\
\hline 11. Is the information in the song enough to help people understand SIT? & $32(80)$ & $52(96)$ \\
\hline \multicolumn{3}{|l|}{ Overall Appropriateness } \\
\hline 12. Is the song appropriate to engage with the community on SIT? & $36(90)$ & $54(100)$ \\
\hline
\end{tabular}


Table 3

Thematic areas and summary on what participants remembered and learned after listening to the song during the assessment of acceptability of a cultural song as a community engagement medium in KwaZulu-Natal Province, South Africa.

\begin{tabular}{|lll|}
\hline Responses from the participants & $\begin{array}{l}\text { Remembered } \\
\mathbf{n = 5 4}(\%)\end{array}$ & $\begin{array}{l}\text { Learned } \\
\mathbf{n}=\mathbf{5 4}(\%)\end{array}$ \\
\hline Female mosquitoes transmit malaria infection & $18(33)$ & $8(15)$ \\
\hline Malaria can kill but is treatable & $13(24)$ & $6(11)$ \\
\hline Male mosquitoes do not bite and do not transmit malaria & $6(11)$ & $13(24)$ \\
\hline Sterilised male mosquito & $3(5)$ & $5(9)$ \\
\hline Medication and indoor residual spraying & $3(5)$ & 0 \\
\hline Signs and symptoms & $2(4)$ & $5(9)$ \\
\hline Warning about malaria & $6(11)$ & $1(2)$ \\
\hline Difference between male and female mosquito behaviour & $3(5)$ & $10(18)$ \\
\hline SIT project & 0 & $3(5)$ \\
\hline Seeking medical treatment if unwell & 0 & $3(5)$ \\
\hline
\end{tabular}

\section{Figures}

\begin{tabular}{|c|c|c|}
\hline $\begin{array}{c}\text { Quantitative data collection } \\
\text { Cross - sectional survey with } \\
\text { community members and } \\
\text { experts } \\
\text { Data analysis }\end{array}$ & $\begin{array}{c}\text { Overall } \\
\text { interpretation of } \\
\text { findings }\end{array}$ \\
\hline
\end{tabular}

Figure 1

Triangulation/ exploratory concurrent mixed methods study design employed to assess acceptability, feasibility and appropriateness of the song in Jozini, KwaZulu-Natal Province, South Africa. 


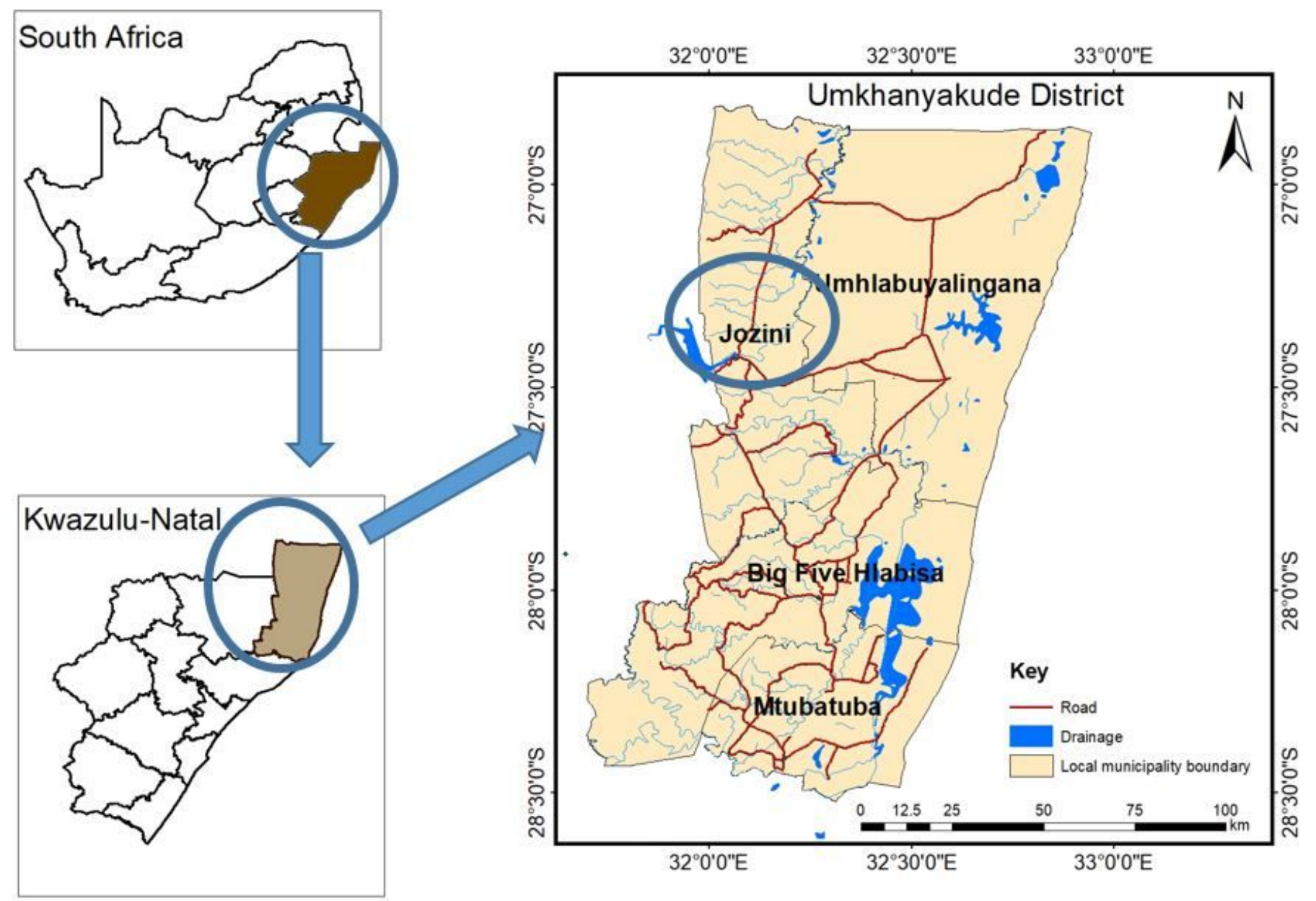

Figure 2

Map of South Africa showing the study area, Mamfene in Jozini local municipality, KwaZulu-Natal Province, South Africa. Note: The designations employed and the presentation of the material on this map do not imply the expression of any opinion whatsoever on the part of Research Square concerning the legal status of any country, territory, city or area or of its authorities, or concerning the delimitation of its frontiers or boundaries. This map has been provided by the authors.

\section{Supplementary Files}

This is a list of supplementary files associated with this preprint. Click to download.

- AdditionalFile1MalariaSongLyrics29102020.docx

- AdditionalFile2Communityquestionnaire.xIsx

- AdditionalFile3Expertsquestionnaire.xIsx 